\title{
Experimental Observation of Single-File Water Filling of Thin Single-Wall Carbon Nanotubes Down to Chiral Index $(5,3)$
}

\author{
Sofie Cambré, Bob Schoeters, Sten Luyckx, Etienne Goovaerts, and Wim Wenseleers* \\ Physics Department, University of Antwerp (Campus Drie Eiken), Universiteitsplein 1, B-2610, Antwerp, Belgium
}

(Received 24 December 2009; revised manuscript received 1 March 2010; published 17 May 2010)

\begin{abstract}
Single-file transport of water into carbon nanotubes is experimentally demonstrated for the first time through the splitting of the radial breathing mode (RBM) vibration in Raman spectra of bile salt solubilized tubes when both empty (closed) and water-filled (open-ended) tubes are present. $\mathrm{D}_{2} \mathrm{O}$ filling is observed for a wide range of diameters, $d$, down to very thin tubes [e.g., $(5,3)$ tube, $d=0.548 \mathrm{~nm}$ ] for which only a single water molecule fits in the cross section of the internal nanotube channel. The shift in RBM frequency upon filling is found to display a very complex dependence on nanotube diameter and chirality, in support of a different yet well-defined ordering and orientation of water molecules at room temperature. Large shifts of the electronic transitions are also observed.
\end{abstract}

DOI: 10.1103/PhysRevLett.104.207401

Transport of water molecules and other liquids through single-wall carbon nanotubes (SWNTs) is a very active area of theoretical research [1,2], in part because of the great potential for applications in nanofluidics [3-6] and ultraselective molecular filtration $[7,8]$. Indeed, SWNTs can have a wide range of very precisely defined diameters and chiral structures, uniquely described by their chiral indices $(n, m)$ [9]. Because of the atomically smooth inner walls, very fast transport of water through SWNTs, orders of magnitude larger than would be expected from macroscopic laws of viscosity, has been predicted theoretically $[1,2]$ and demonstrated experimentally [7]. Extending this to thin SWNTs, extreme selectivity at the molecular scale would be achievable, reminiscent of the selective transport of molecules through cell membranes via porine proteins [10]. In addition, SWNTs have unique electronic and optical properties, also depending critically on the chiral structure, with tubes being either metallic or semiconducting. This offers interesting opportunities for the fabrication of nanoelectro-fluidic devices such as nanopumps [11] or hydroelectric power converters [12]. Water filling of very thin SWNTs is also very appealing from a fundamental point of view, as this would create the ideal conditions to achieve a single-file "first-in-first-out" transport regime, even over long distances and time scales (SWNTs can be up to centimeters long [13])—phenomena which are also important to the understanding of diffusion in zeolites [14]. In SWNTs thinner than about $0.95 \mathrm{~nm}$, mutual passage of water molecules would be excluded [15] and strongly correlated molecular motion is predicted to occur $[16,17]$. Theoretical calculations (mostly only classical, and limited to nonchiral SWNTs $[2,18]$ ) have predicted water filling of SWNTs down to a chiral index of about $(5,5)(d=0.678 \mathrm{~nm})$ [15]. However, experimental evidence for water filling of carbon nanotubes [19] is only available for larger diameters. Microscopy techniques (optical [20] or electron microscopy [21]) can probe water filling in multiwall and thick SWNTs, while spectroscopic
PACS numbers: 78.30.Na, 47.61.Cb, 66.30.Pa, 78.67.Ch

studies of the water filling in SWNTs (x-ray scattering [22], neutron scattering [23], NMR [24], IR-spectroscopy [25], and Raman on tangential modes of SWNTs [26]) yield an ensemble average of the many different diameters and chiralities present in the bulk samples used. Theoretical work has predicted that also the radial breathing mode (RBM) frequency of the SWNTs themselves changes upon water filling [27]. Recently, we have experimentally demonstrated that it is indeed possible to detect this shift [28], for SWNTs with a diameter of $d \sim 1 \mathrm{~nm}$, provided the SWNTs are solubilized in water using bile salt surfactants [29], which dramatically increases the spectral resolution due to the individual isolation of the tubes in a very homogeneous micelle layer. In this Letter, we report on the experimental observation of water filling of SWNTs for a wide range of SWNT structures, down to extremely thin diameters $\{(5,3), d=0.548 \mathrm{~nm}[30]\}$, the internal channel of which is only just wide enough to fit a single water molecule. Using resonant Raman scattering, we are able to study the water filling selectively for each specific SWNT structure separately, which is a major advantage compared to other experimental techniques [19].

In order to have a sufficient concentration of thin tubes, SWNTs synthesized using either the high pressure CO decomposition method [31] $(d=1.05 \pm 0.35 \mathrm{~nm}$; HipCO1: raw, as-produced material; HipCO2: purified) or the cobalt molybdenum catalyst method [32] $(d=$ $0.8 \pm 0.1 \mathrm{~nm}$; CoMoCAT SG65 grade) were used in this study [18]. These samples were solubilized in $\mathrm{D}_{2} \mathrm{O}$ using the bile salt surfactant sodium deoxycholate [29] and centrifuged as described in [18]. $\mathrm{D}_{2} \mathrm{O}$ is used throughout this study, as usual, as the higher density yields an optimal separation of individual tubes from bundles during centrifugation [33]. The as-produced material (HipCO1) consists largely of closed (end-capped) SWNTs, which are partly opened (cut) using ultrasonication [18]. The other two SWNT materials have been chemically purified, which results in nearly all of the tubes being opened (the actual 
percentage of opening depending on the SWNT structure and/or defect density). The solutions were investigated by resonant Raman spectroscopy using 46 different wavelengths from three lasers $\left(\mathrm{Ar}^{+}, \mathrm{Kr}^{+}\right.$and Ti:sapphire laser) ranging from $406.7 \mathrm{~nm}$ to $865 \mathrm{~nm}$. For the $(n, m)$ assignment, the measured RBM frequencies and respective excitation energies are compared to empirical relations from literature for semiconducting [33,34] and metallic [35] tubes.

For each SWNT structure in electronic resonance with the given laser wavelength, two RBM peaks are observed in the Raman spectrum. In our previous work on largerdiameter SWNTs [28], we have shown that such a bimodal distribution can only be explained by the presence of empty (end-capped) and water-filled (opened) SWNTs. The reasoning and additional test experiments to exclude any alternative explanations [28] are the same for these thinner tubes [18]. Both peaks can be very accurately fitted with Lorentzians, through a simultaneous curve fit of spectra of different samples, containing different fractions of opened (water-filled) tubes. To exclude that any of the additional peaks would arise from filling with other molecules, such as $\mathrm{O}_{2}$ or $\mathrm{N}_{2}$, measurements were also performed on samples prepared in vacuum [18]. However, the same results were obtained. For nearly all SWNTs [including the thinnest, $(5,3)$ tube], samples with $\sim 100 \%$ water filling were observed, indicating that the tubes, when opened, are completely filled. Conversely, the observation of empty tubes in all of the pristine samples implies that a significant fraction of the tubes is capped at both ends and, over the entire length of the tubes, completely free of defects large enough to pass a water molecule. The graphene sidewalls of SWNTs are perfectly impermeable to water (or any other molecules or atoms) [36].

Spectra obtained at an excitation wavelength of $568.2 \mathrm{~nm}$ are shown in Fig. 1. A first interesting observation is that the RBM frequency shift induced by the water filling is very different for different SWNT structures, as is the filling-induced broadening of the vibrational resonance. We obtained these RBM shifts for 36 different SWNT chiralities within the diameter range of 0.548 to $1.230 \mathrm{~nm}$, which are plotted in Fig. 2 [18]. This reveals a very complex, nonmonotonic dependence of the RBM shift on tube diameter and chiral structure. While the RBM shifts are in the order of magnitude of theoretical predictions based on a continuum model [27], the complex experimentally observed behavior obviously cannot be described by such a model. This should not surprise, as the cross section of the internal channel of the tubes considered here ranges from about one to two times the size of a water molecule. The general observations are in line with the predictions of well-defined ordered ("solidlike") structures formed by water molecules at room temperature when confined in nanotubes $[2,17,37]$. While the experimental data show no obvious overall trend with diameter, metallic SWNTs generally display larger RBM shifts upon filling than semiconducting tubes having simi-

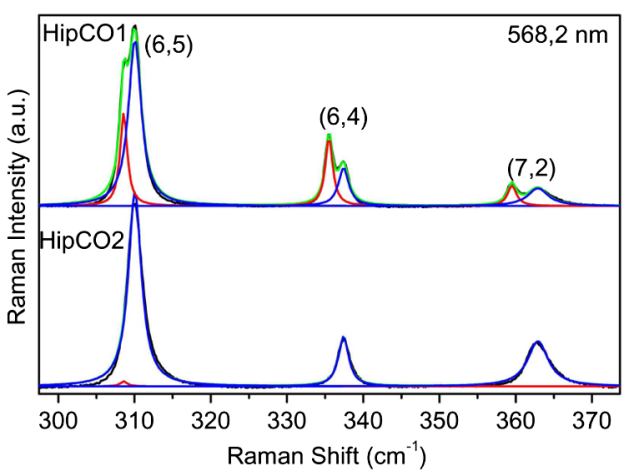

FIG. 1 (color online). Resonant Raman spectra for partially closed (top) and largely opened (bottom) HipCO samples, excited at $568.2 \mathrm{~nm}$, showing resonances for $(6,5),(6,4)$, and $(7,2)$ tubes. Experimental spectra (black line) are shown together with a best fit [light gray (green) line] composed of Lorentzian components for empty [closed; gray (red)] and $\mathrm{D}_{2} \mathrm{O}$-filled [opened; dark gray (blue)] tubes.

lar diameters. Possibly, the higher polarizability of metallic tubes results in a better wetting of these tubes, as was also concluded from theoretical calculations on the external adsorption of water [38]. Furthermore, from the experimental data, it appears that also among the semiconducting tubes, a slightly larger RBM shift occurs for tubes with $(n-m) \bmod 3=2$ than for similar diameters with a modulus of 1. Extending the above argument from Ref. [38], this may indicate that the semiconducting mod 1 tubes possess the least wettable inside surface. Clearly, the present data show that the RBM shift not only depends on the SWNT diameter, but also on its chirality. This is particularly well illustrated by the pairs of SWNT structures, $(6,5)$ and $(9,1)$, and $(11,1)$ and $(9,4)$, which, in the standard geometrical model [30] have exactly the same diameter but show significantly different shifts of the RBM upon water filling. This effect may be due to a purely

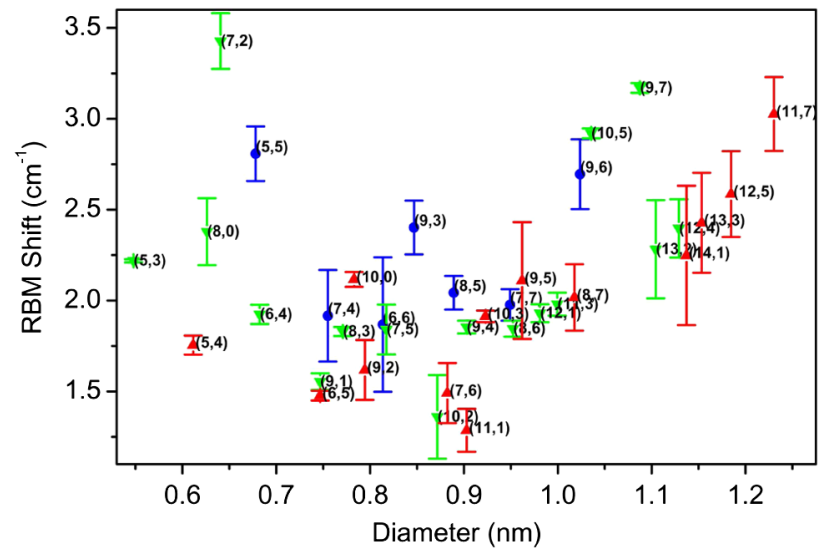

FIG. 2 (color online). Radial breathing mode (RBM) frequency shift upon $\mathrm{D}_{2} \mathrm{O}$ filling for a range of single-walled carbon nanotubes, plotted as a function of nanotube diameter. Symbols indicate metallic tubes $[(n-m) \bmod 3=0$; blue circles $]$, and semiconducting tubes with $(n-m) \bmod 3=1$ (upward red triangles) and $(n-m) \bmod 3=2$ (downward green triangles). 
geometrical influence-the chiral angle changing the SWNT unit cell length and influencing the best fitting water spiral structure - or may result indirectly through a difference in electronic properties. Indeed, while all of these four tubes are semiconducting, they do have different $(n-m)$ mod 3 values.

Both the shift and broadening are largest for the $(7,2)$ tube, with a diameter of $0.641 \mathrm{~nm}$, well within the range for which only a single file of water molecules fits in the tube [15]. Surprisingly, the even thinner $(8,0),(5,4)$, and $(5,3)$ tubes (which still display water filling, and thus a tighter fit of the molecules in the channel is expected) show a smaller RBM shift than the $(7,2)$ tube. This indicates a different orientation of the water molecules in $(8,0),(5,4)$, and $(5,3)$ tubes than in $(7,2)$ tubes the long axis of the water molecules, i.e., the $\mathrm{H}-\mathrm{H}$ connecting vector being parallel to the tube axis for the thinnest tubes $[(8,0)$ through $(5,3)]$. We also confirmed these different orientations by semiempirical calculations using the PM6 Hamiltonian [18]. For thicker tubes, theoretical prediction of the most stable structure of the water molecules formed becomes far more challenging as the additional room allows for many more degrees of freedom, resulting in complex single or multiple spiral structures $[2,15,37]$. Further work will be required to model these "ice nanotube" structures for each chirality, and to link them to the RBM shifts observed here, providing a critical test for the theoretical models.

In order to also investigate the influence on the electronic structure of the SWNTs, 2D (i.e., excitation wavelength dependent) Raman maps were recorded for the very thin $(5,3)$ and $(5,4)$ tubes throughout the wavelength range of their lowest energy transition $\left(E_{11}\right)$ (Fig. 3). This transition energy is found to shift very strongly for the $(5,4)$ tube, from $1.495 \mathrm{eV}$ (empty) to $1.457 \mathrm{eV}$ (water-filled) $\left(\Delta E_{11}=38.1 \mathrm{meV}\right.$ [18]). Preliminary results also indicate a large shift in the second optical transition [18]. This shift
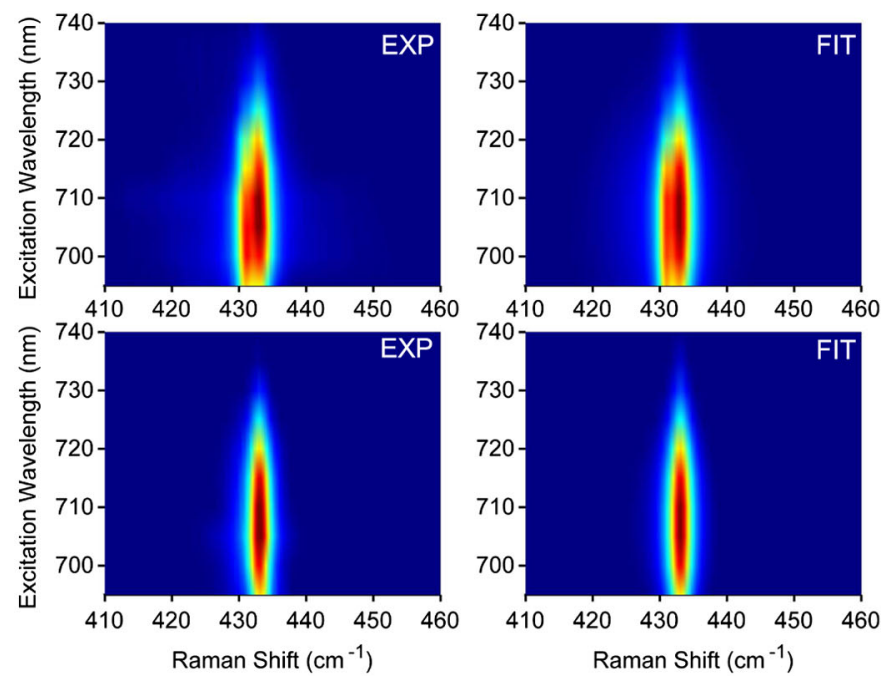

is much larger than for the thinner $(5,3)$ tube $\left(\Delta E_{11}=\right.$ $9.9 \mathrm{meV}$ ) and also much larger than the previously observed shift of the second electronic transition of $\sim 1 \mathrm{~nm}$ thick tubes [28] $\left(\Delta E_{22}=7.1-10.7 \mathrm{meV}\right)$. This is quite important in view of the fact that empirical data for the electronic transition energies of SWNTs in literature are mainly based on spectroscopic measurements on aqueous solutions of heavily ultrasonicated, and therefore largely opened SWNTs [33]. The large differences in the electronic shifts for different SWNTs may also be linked to differences in orientation of the water molecules.

The observation that all SWNTs, down to the very thinnest SWNTs observed, get filled with water when opened is also very important to the separation and purification of SWNTs by ultracentrifugation. Indeed, since our discovery that SWNTs can be efficiently and individually solubilized using bile salts (also known as steroid surfactants or cholates) [29], huge progress in SWNT separation techniques was achieved, and, in particular, the macroscopic sorting of SWNTs according to diameter, electronic type, and even chiralities and handedness (thanks to the chiral nature of bile salts) was realized by density gradient ultracentrifugation (DGU) [39]. Importantly, the complex, nonmonotonic behavior seen in Fig. 2 (and the underlying complexity of the water structure in the tubes), indicates that also the effective density of the filled tubes, according to which they are sorted in DGU, may follow a nonmonotonic dependence on the diameter. As a result, better separation may be expected when using pristine, closed tubes.

The extensive dataset obtained for both empty and water-filled SWNTs also allows us to test or improve models for the dependence of RBM frequency on diameter $d[30]$ and environment. For a long time, the dependence of $\omega_{\mathrm{RBM}}$ on $d$ was modeled by $\omega_{\mathrm{RBM}}=A / d+B$ [33], for which many different values of $A$ and $B$ can be found in literature. Recently, it was pointed out that the constant $B$ is
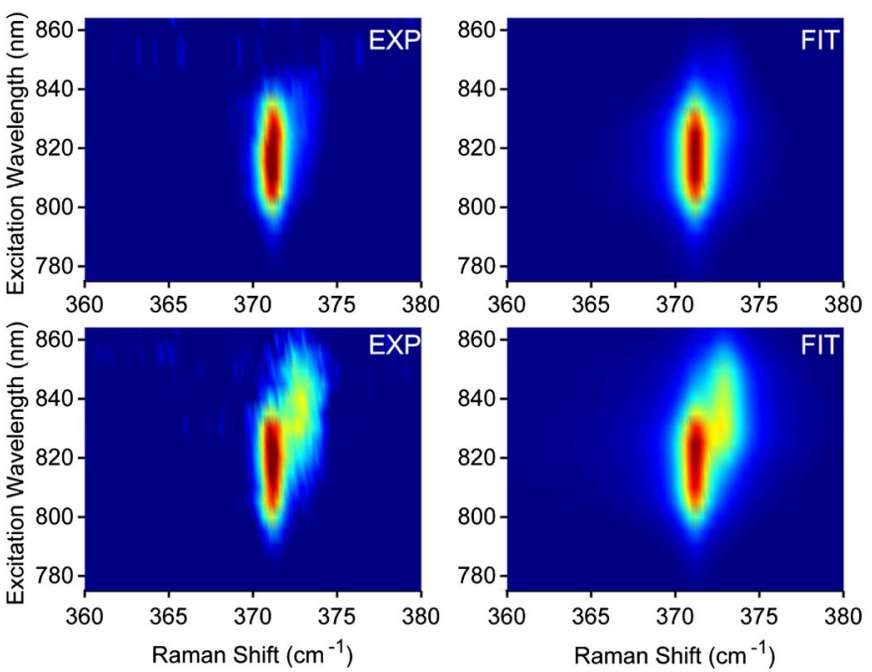

FIG. 3 (color online). Two-dimensional Raman-excitation maps through the first electronic transition of the (5,3) tube (left), and $(5,4)$ tube (right) for HipCO1 (top) and HipCO2 (bottom). The sample used for the upper panes contains less open (filled) tubes than the sample used for the lower ones. The best fit is shown to the right of each experimental map. 
not needed for SWNTs in vacuum [35], but had to be introduced due to interactions with the surrounding environment for SWNTs in solution. A more physical model accounting for such environment effects resulted in an alternative form: $\omega_{\mathrm{RBM}}=\left(227 \mathrm{~cm}^{-1} \mathrm{~nm} / d\right) \sqrt{1+C d^{2}}$ [40]. We fitted our data for both the empty and water-filled SWNTs with both models, and compared these to most commonly used sets of parameters [18]. In agreement with the general idea that the second parameter $(B$ or $C$ ) describes the interaction with environment, we find a larger deviation from the simple reciprocal dependence (larger values of $B$ or $C$ ) for the water-filled SWNTs (interaction with external surfactant layer and with internal water; $C=$ $0.073 \mathrm{~nm}^{-2}$ ) than for the empty SWNTs (interaction with external environment only; $C=0.053 \mathrm{~nm}^{-2}$ ) [18]. Further work, over a range including larger diameters as well, would be needed to validate either of both models. While both models fit the overall trend with $d$ well, residual deviations are significant [18] and show no obvious correlation with diameter or chiral angle, which may be ascribed to a different ordering of the surfactant molecules on the SWNTs.

In summary, water filling is observed for 36 different SWNT chiralities $(n, m)$ over a wide diameter range $(0.548$ to $1.230 \mathrm{~nm}$ ), through the shifts of the RBM frequency upon water filling, showing a complex nonmonotonic dependence on the diameter and chirality and demonstrating water filling of tubes well in the diameter range where only a single file of water molecules fits inside the tube. This is extremely promising in view of nanofluidics and ultrafiltration applications, but also relevant to the DGU separation of SWNTs. Filling of SWNTs may also dramatically improve their mechanical resistance to high pressures [41]. Largest RBM shifts were observed for the $(7,2)$ SWNT, attributed to different orientations of the water molecules in these than in the thinner tubes. Also, large electronic shifts were observed upon water filling. These results also provide detailed experimental information to critically test theoretical models for water transport through SWNTs.

Financial support from the Fund for Scientific Research Flanders, Belgium (FWO-Vlaanderen) (Project No. G.0129.07), is gratefully acknowledged.

*Wim.Wenseleers@ua.ac.be

[1] G. Hummer, J. C. Rasaiah, and J. P. Noworyta, Nature (London) 414, 188 (2001).

[2] A. Alexiadis and S. Kassinos, Chem. Rev. 108, 5014 (2008), and references therein.

[3] C. Dellago and G. Hummer, Phys. Rev. Lett. 97, 245901 (2006).

[4] J. K. Holt, Adv. Mater. 21, 3542 (2009).

[5] A. Noy et al., Nano Today 2, 22 (2007).

[6] J. C. T. Eijkel and A. van den Berg, Microfluid. Nanofluid. 1, 249 (2005).

[7] J. K. Holt et al., Science 312, 1034 (2006); Y. Maniwa et al., Nature Mater. 6, 135 (2007).
[8] B. Corry, J. Phys. Chem. B 112, 1427 (2008).

[9] S. Reich, C. Thompson, and J. Maultzsch, Carbon Nanotubes: Basic Concepts and Physical Properties (Wiley-VCH, Berlin, 2004).

[10] P. Agre et al., J. Physiol. 542, 3 (2002).

[11] X. Gong et al., Nature Nanotech. 2, 709 (2007).

[12] Y. C. Zhao et al., Adv. Mater. 20, 1772 (2008).

[13] L. X. Zheng et al., Nature Mater. 3, 673 (2004).

[14] B. Smit and T. L. M. Maesen, Chem. Rev. 108, 4125 (2008); K. Hahn, J. Karger, and V. Kukla, Phys. Rev. Lett. 76, 2762 (1996).

[15] J. Wang et al., Phys. Chem. Chem. Phys. 6, 829 (2004); L. Y. Meng, Q. K. Li, and Z. G. Shuai, J. Chem. Phys. 128, 134703 (2008).

[16] B. Mukherjee et al., J. Chem. Phys. 126, 124704 (2007).

[17] J. A. Thomas and A. J.H. McGaughey, Phys. Rev. Lett. 102, 184502 (2009).

[18] See supplementary material at http://link.aps.org/ supplemental/10.1103/PhysRevLett.104.207401 for additional discussion, experimental and theoretical details, a selection of fits, and table of data: EPAPS.pdf and EPAPS.xls.

[19] J. Holt, Microfluid. Nanofluid. 5, 425 (2008).

[20] B. M. Kim, S. Sinha, and H. H. Bau, Nano Lett. 4, 2203 (2004).

[21] D. Mattia and Y. Gogotsi, Microfluid. Nanofluid. 5, 289 (2008); N. Naguib et al., Nano Lett. 4, 2237 (2004).

[22] Y. Maniwa et al., J. Phys. Soc. Jpn. 71, 2863 (2002); Chem. Phys. Lett. 401, 534 (2005).

[23] N. R. de Souza et al., J. Phys. Condens. Matter 18, S2321 (2006); A. I. Kolesnikov et al., Phys. Rev. Lett. 93, 035503 (2004).

[24] S. Ghosh, K. V. Ramanathan, and A. K. Sood, Europhys. Lett. 65, 678 (2004); W. Sekhaneh et al., Chem. Phys. Lett. 428, 143 (2006); K. Matsuda et al., Phys. Rev. B 74, 073415 (2006); H. J. Wang et al., Science 322, 80 (2008); Q. Chen et al., Nano Lett. 8, 1902 (2008).

[25] O. Byl et al., J. Am. Chem. Soc. 128, 12090 (2006).

[26] S. C. Sharma, D. Sing, and Y. Li, J. Raman Spectrosc. 36, 755 (2005).

[27] M. J. Longhurst and N. Quirke, J. Chem. Phys. 125, 184705 (2006).

[28] W. Wenseleers et al., Adv. Mater. 19, 2274 (2007).

[29] W. Wenseleers et al., Adv. Funct. Mater. 14, 1105 (2004).

[30] Diameters were calculated using a fixed carbon-carbon distance of $d_{\mathrm{cc}}=0.142 \mathrm{~nm}$.

[31] P. Nikolaev et al., Chem. Phys. Lett. 313, 91 (1999).

[32] B. Kitiyanan et al., Chem. Phys. Lett. 317, 497 (2000).

[33] S. M. Bachilo et al., Science 298, 2361 (2002).

[34] P. T. Araujo et al., Phys. Rev. Lett. 98, 067401 (2007).

[35] S. K. Doorn et al., Phys. Rev. B 78, 165408 (2008).

[36] O. Leenaerts, B. Partoens, and F. M. Peeters, Appl. Phys. Lett. 93, 193107 (2008).

[37] K. Koga et al., Nature (London) 412, 802 (2001).

[38] M. J. Longhurst and N. Quirke, Phys. Rev. Lett. 98, 145503 (2007).

[39] M.S. Arnold et al., Nature Nanotech. 1, 60 (2006); A. A. Green, M. C. Duch, and M.C. Hersam, Nano Res. 2, 69 (2009); F. Hennrich, S. Lebedkin, and M. M. Kappes, Phys. Status Solidi B 245, 1951 (2008).

[40] P. T. Araujo et al., Phys. Rev. Lett. 103, 146802 (2009).

[41] A. Merlen et al., Phys. Rev. B 72, 035409 (2005). 